\title{
Semi-urgent pulmonary vein isolation using cryoballoon for haemodynamically unstable atrial fibrillation storm in a patient with low cardiac output syndrome: a case report
}

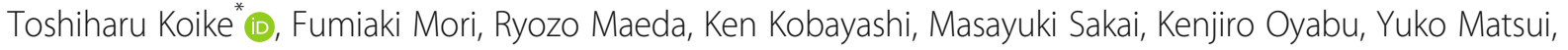
Masafumi Yoshikawa, Kou Sugiyama, Yoichi Ajiro and Kazunori Iwade

\begin{abstract}
Background: Atrial fibrillation and heart failure are common coexisting conditions requiring hospitalisation for heart failure and death. Pulmonary vein isolation is a well-established option for symptomatic atrial fibrillation and for atrial fibrillation concomitant with heart failure with reduced left ventricular ejection fraction. Recently, pulmonary vein isolation using cryoballoon showed non-inferiority to radiofrequency ablation with respect to the treatment of patients with drug-refractory paroxysmal atrial fibrillation. However, the effectiveness of acute-phase rhythm control by semi-urgent pulmonary vein isolation using cryoballoon in patients with haemodynamically unstable atrial fibrillation storm accompanied with low cardiac output syndrome is unclear. Herein, we present a case in which semi-urgent pulmonary vein isolation using cryoballoon was effective for acute-phase rhythm control against drug-resistant and haemodynamically unstable repetitive atrial fibrillation tachycardia accompanied with low cardiac output syndrome.
\end{abstract}

Case presentation: A 57-year-old man was hospitalised for New York Heart Association functional class 4 heart failure with atrial fibrillation tachycardia and reduced left ventricular ejection fraction of $20 \%$ accompanied with low cardiac output syndrome-induced liver damage. The haemodynamics collapsed during atrial fibrillation tachycardia, which had become resistant to intravenous amiodarone and repeated electrical cardioversions. In addition to atrial fibrillation, atrial tachycardia and common-type atrial flutter appeared on day 3. Multiple organ failure progressed gradually due to haemodynamically unstable atrial fibrillation tachycardia storm accompanied with low cardiac output syndrome. On day 4, to focus on treatment of heart failure and multiple organ failure, semi-urgent rescue pulmonary vein isolation using cryoballoon to atrial fibrillation and cavotricuspid isthmus ablation to common-type atrial flutter were performed for acute-phase rhythm control. Soon after the ablation procedure, atrial fibrillation and common-type atrial flutter were lessened, and sinus rhythm was restored. A stable haemodynamics was successfully achieved with the improvement of hepatorenal function. The patient was discharged on day 77 without complications.

(Continued on next page)

\footnotetext{
* Correspondence: ffayf195@yahoo.co.jp

Department of Cardiology, National Hospital Organization Yokohama Medical Center, 3-60-2 Harajuku, Totsuka-ku, Yokohama-shi, Kanagawa 245-8575, Japan
}

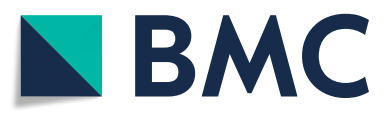

(c) The Author(s). 2020 Open Access This article is licensed under a Creative Commons Attribution 4.0 International License, which permits use, sharing, adaptation, distribution and reproduction in any medium or format, as long as you give appropriate credit to the original author(s) and the source, provide a link to the Creative Commons licence, and indicate if changes were made. The images or other third party material in this article are included in the article's Creative Commons licence, unless indicated otherwise in a credit line to the material. If material is not included in the article's Creative Commons licence and your intended use is not permitted by statutory regulation or exceeds the permitted use, you will need to obtain permission directly from the copyright holder. To view a copy of this licence, visit http://creativecommons.org/licenses/by/4.0/. The Creative Commons Public Domain Dedication waiver (http://creativecommons.org/publicdomain/zero/1.0/) applies to the data made available in this article, unless otherwise stated in a credit line to the data. 
(Continued from previous page)

Conclusions: This case demonstrates that acute-phase rhythm control by semi-urgent pulmonary vein isolation using cryoballoon could be a treatment option in patients with haemodynamically unstable atrial fibrillation tachycardia storm accompanied with low cardiac output syndrome, which is refractory to cardioversion and drug therapy.

Keywords: Atrial fibrillation, Low cardiac output syndrome, Catheter ablation, Congestive heart failure, Pulmonary vein isolation, Cryoballoon ablation

\section{Background}

Atrial fibrillation (AF) and heart failure (HF) are common coexisting conditions with hospitalisation for HF and death [1]. Pulmonary vein isolation (PVI) is a well-established option for symptomatic paroxysmal AF [2] and for AF concomitant with HF with reduced left ventricular ejection fraction (HFrEF) [3-6]. PVI for patients with AF concomitant with HFrEF demonstrated significant reduction in overall mortality rate and incidence of hospitalisation for worsening $\mathrm{HF}$ and improvement in left ventricular ejection fraction (LVEF) compared with the conventional drug therapy [5]. The cryoballoon is a recently developing ablation tool that showed non-inferior efficacy and overall safety [7-9]. However, the role and significance of acute-phase rhythm control by semi-urgent PVI is not yet established. Herein, we present a case in which semi-urgent PVI using cryoballoon was effective for acute-phase rhythm control against drugresistant and haemodynamically unstable repetitive AF tachycardia storm accompanied with low cardiac output syndrome (LOS) and LOS-induced multiple organ failure (MOF).

\section{Case presentation}

A 57-year-old man was referred to our hospital for New York Heart Association functional class $4 \mathrm{HF}$ and extremely elevated liver enzymes. He had palpitation from 2 weeks ago which was accompanied with orthopnoea and serious fatigue on admission, despite being healthy without any history of AF. The electrocardiography showed AF tachycardia of approximately 180 beats per minute, and the bedside echocardiography showed low LVEF of $20 \%$. In addition to fatigue, coexisting hypotension and elevated lactate of $13 \mathrm{mmol} / \mathrm{L}$ indicated LOS. Electrical cardioversion was conducted, barely terminated AF successfully on day 1 , and restored blood pressure and urinalysis response to intravenous furosemide. Intravenous landiolol hydrochloride was administered for AF tachycardia. HF and LOS were treated with intravenous dobutamine, intravenous furosemide and oral tolvaptan, and non-invasive positive pressure ventilation under mild sedation using intravenous dexmedetomidine hydrochloride. On day 2, torsade de pointes suddenly occurred subsequent to a premature atrial beat in a long-short manner of coupling interval with QT prolongation. Cardiopulmonary resuscitation with electric cardioversions, intratracheal intubation, and establishment of mechanical ventilation were carried out, which achieved return of spontaneous circulation. Temporary atrium-atrium inhibited pacing was emergently established to shorten prolonged QT and maintain regular heart rate. However, AF tachycardia recurred repetitively. Repeated electrical cardioversions failed to terminate AF. Hypotension continued along with oliguria. The liver dysfunction was further exacerbated with aspartate aminotransferase of $11,708 \mathrm{U} / \mathrm{L}$. The intravenous amiodarone was started. On day 3, AF tachycardia with hypotension still occurred despite intravenous amiodarone and gradually became resistant to electrical cardioversions. The atrial tachycardia (AT) and common-type atrial flutter (AFL) appeared in addition to AF. On day 4, disseminated intravascular coagulation (DIC) was diagnosed according to the Japanese Society on Thrombosis and Hemostasis criteria, with DIC score of 6 points [10]. Acute kidney injury was diagnosed according to the Kidney Disease Improving Global Outcomes criteria, fulfilling both serum creatine and urine output criteria [11]. At this time, because the patient's general condition was becoming worse and more resistant to treatment, we decided to perform semi-urgent rescue ablation to AF and common-type AFL for acute-phase rhythm control to treat $\mathrm{HF}$ and MOF.

The patient's family agreed with our treatment policy and signed informed consent for the semi-urgent rescue ablation procedure.

On the same day, prior to the procedure, transoesophageal echocardiography was performed and found no detectable thrombus in the left atrium (LA). PVI to the AF and cavotricuspid isthmus ablation to commontype AFL were planned. Fortunately, the patient's haemodynamics did not collapse at the time of ablation, we performed our institutional standard procedure including 3-D mapping. The cryoballoon was chosen as a PVI catheter because cryoballoon ablation was expected 
to have a shorter procedure time and lesser thrombogenic effect than other catheter types [7, 12]. A 20-polar catheter (Response ${ }^{\mathrm{Tm}}$; Abbott, St. Paul, MN, USA) was placed in the coronary sinus. A three-dimensional electroanatomical mapping was constituted by a cardiac mapping system (EnSite velocity ${ }^{\text {tix }}$; Abbott). Transseptal access was obtained using the standard Brockenbrough needle technique with intracardiac ultrasound and fluoroscopic guidance, and an 8-Fr SL0 sheath (Swartz ${ }^{\mathrm{mm}}$; Abbott) was inserted into the LA. The cardiac geometry including all pulmonary veins (PV) was established using a 20-pole circular mapping catheter (Reflexion Spiral ${ }^{\mathrm{ma}}$; Abbott). No left atriography was conducted because of acute kidney injury. Then, we changed the SL0 sheath to a steerable sheath (FlexCath Advance ${ }^{\mathrm{Tw}}$; Medtronic Inc., Minneapolis, MN, USA) and inserted a $28-\mathrm{mm}$ secondgeneration cryoballoon catheter (Arctic Front Advance $^{\text {tw }}$; Medtronic Inc.). In each PV, the cryoballoon was placed at the ostium of each PV in turns, and cryoballoon ablation was performed after complete occlusion of each PV as confirmed by the minimum amount of contrast agent. During cryoenergy deliveries, the oesophageal temperature and diaphragmatic compound motor action potential were monitored to avoid LA-oesophageal fistula and phrenic nerve injury. Additional touch-up radiofrequency ablation using FlexAbility $^{\text {ma }}$ (Abbott) to the residual LA-PV conduction gap at the bottom of the right inferior PV after cryoballoon ablation was conducted, and complete PVI was achieved (detailed PVI procedural data is shown in Table 1 and Fig. 1). Subsequently, a 20- polar catheter (Livewire ${ }^{\mathrm{m}}$; Abbott) was placed around the tricuspid annulus, confirming that the AFL was cavotricuspid-isthmus dependent. The cavotricuspid isthmus ablation for common-type AFL was performed by standard procedure using radiofrequency ablation catheter and successfully achieved bidirectional block. The whole procedure was finished uneventfully and restored sinus rhythm and blood pressure of approximately $110 \mathrm{mmHg}$.

Thereafter, AF and other atrial arrhythmias seldom occurred and was terminated by single electrical cardioversion (Fig. 2). Normal blood pressure and urine output were restored (Fig. 2). The hepatic and renal functions were improved gradually as well. On day 7 , intravenous amiodarone was discontinued. On day 11, the patient was weaned from the ventilator support. The DIC was restored eventually. On day 18, pulmonary abscess that required long-term antimicrobial treatment was cured. On day 22, AF recurred, but oral amiodarone was restarted and suppressed the AF. Although the patient had a pulmonary abscess that required approximately one-month antimicrobial treatment after ventilator withdrawal, he was discharged alive on day 72 . The elective coronary angiography and left ventriculography revealed no significant coronary stenosis and LVEF normalisation, diagnosing that the tachycardia-induced cardiomyopathy due to AF tachycardia was the cause of reduced left ventricular function. For 2 years, albeit the discontinuation of HF drugs and amiodarone, the patient has been free from HF symptoms and atrial arrhythmias including AF and AFL.

Table 1 The procedural data of pulmonary vein isolation using cryoballoon

\begin{tabular}{rrrrr}
\hline Total procedure time (minute) & 164 & & & \\
Fluoroscopic time (minute) & 97 & & & \\
Fluorocopic dose (gray) & 2.4 & & & \\
& LSPV & LIPV & RSPV & RIPV \\
Freeze durarion (second) & 180 & 252 & 360 & 180 \\
Time to isolation (second) & Not recorded Not recorded Not recorded & Not recorded \\
Total freeze (times) & 1 & 2 & 2 & 1 \\
Minimal freeze teperature $\left({ }^{\circ} \mathrm{C}\right)$ & -56 & -47 & -52 & -48 \\
Occulusion grade & OG3 & OG3 & OG3 & OG3 \\
\hline
\end{tabular}

The occlusion grade (OG) was scored as follows: OG3 (complete occlusion), OG2 (incomplete occlusion with slight leakage), OG1 (poor occlusion with massive leakage)

LSPV left superior pulmonary vein, LIPV left inferior pulmonary vein, RSPV right superior pulmonary vein, RIPV right inferior pulmonary vein, OG Occlusion grade 


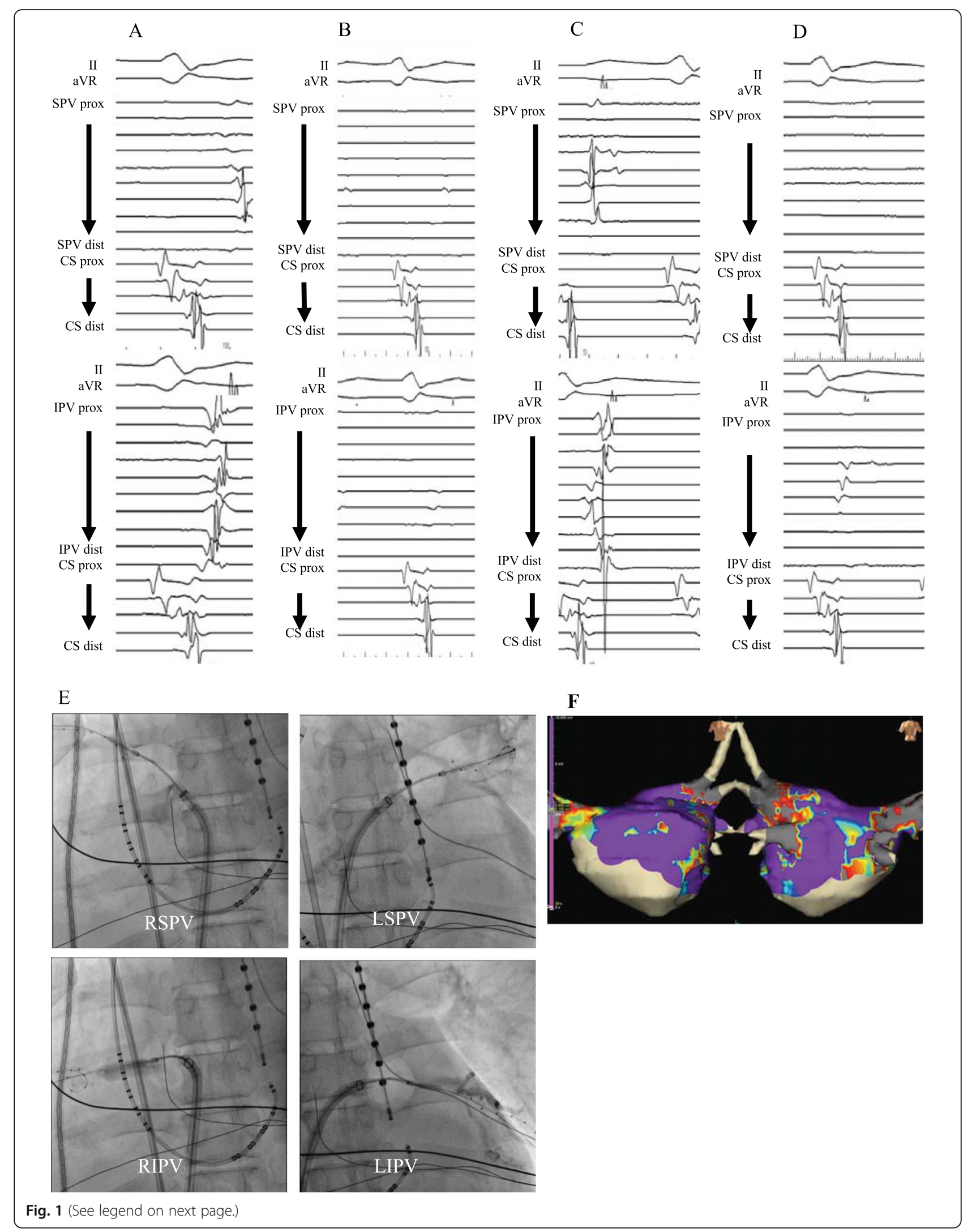


(See figure on previous page.)

Fig. 1 Pulmonary vein isolation using cryoballoon. a: Baseline intracardiac electrograms of the left pulmonary veins before isolation. b: Intracardiac electrograms after the left pulmonary vein isolation. c: Baseline intracardiac electrograms of the right pulmonary veins before isolation. d: Intracardiac electrograms after the right pulmonary vein isolation. e: Fluoroscopic AP image demonstrating positions of the cryoballoon for all pulmonary veins. f: Three-dimensional map with grey area representing ablated area by pulmonary vein isolation using cryoballoon. CS, coronary sinus; dist, distal bipole; prox, proximal bipole; IPV, inferior pulmonary vein; LIPV, left inferior pulmonary vein; LSPV, left superior pulmonary vein; RIPV, right inferior pulmonary vein; RSPV, right superior pulmonary vein; SPV, superior pulmonary vein

\section{Discussion and conclusion}

To our knowledge, this is the first report of semi-urgent rescue PVI using cryoballoon for acute-phase rhythm control against amiodarone-resistant AF tachycardia storm causing LOS and LOS-induced MOF with DIC in a tachycardia-induced cardiomyopathy.

AF may cause adverse haemodynamic effects and lead to decrease in cardiac output through the loss of atrial contraction, reduction of left ventricular filling due to rapid ventricular rates and irregular RR interval, increase maximal oxygen consumption, and exacerbate mitral and tricuspid regurgitation [1]. Therefore, restoration of sinus rhythm in AF patients can be expected to improve cardiac output and decrease maximal oxygen consumption [3, 4]. PVI is the established treatment for the rhythm control strategy of AF even refractory to antiarrhythmic drugs [2] because PVI has an anti-AF mechanism different from that of drugs, such as eliminating AF substrate, denervating the autonomic nerve, and most importantly eliminating AF triggers arising from PVs $[13,14]$. On the contrary, the role and significance of acute-phase rhythm control by semi-urgent PVI is not yet established. However, Morishima et al. reported a similar case. They described that the semiurgent rescue PVI could eliminate haemodynamically unstable AF storm and contribute to the improvement of haemodynamic states in a patient with an acute myocardial infarction, although the patient died from

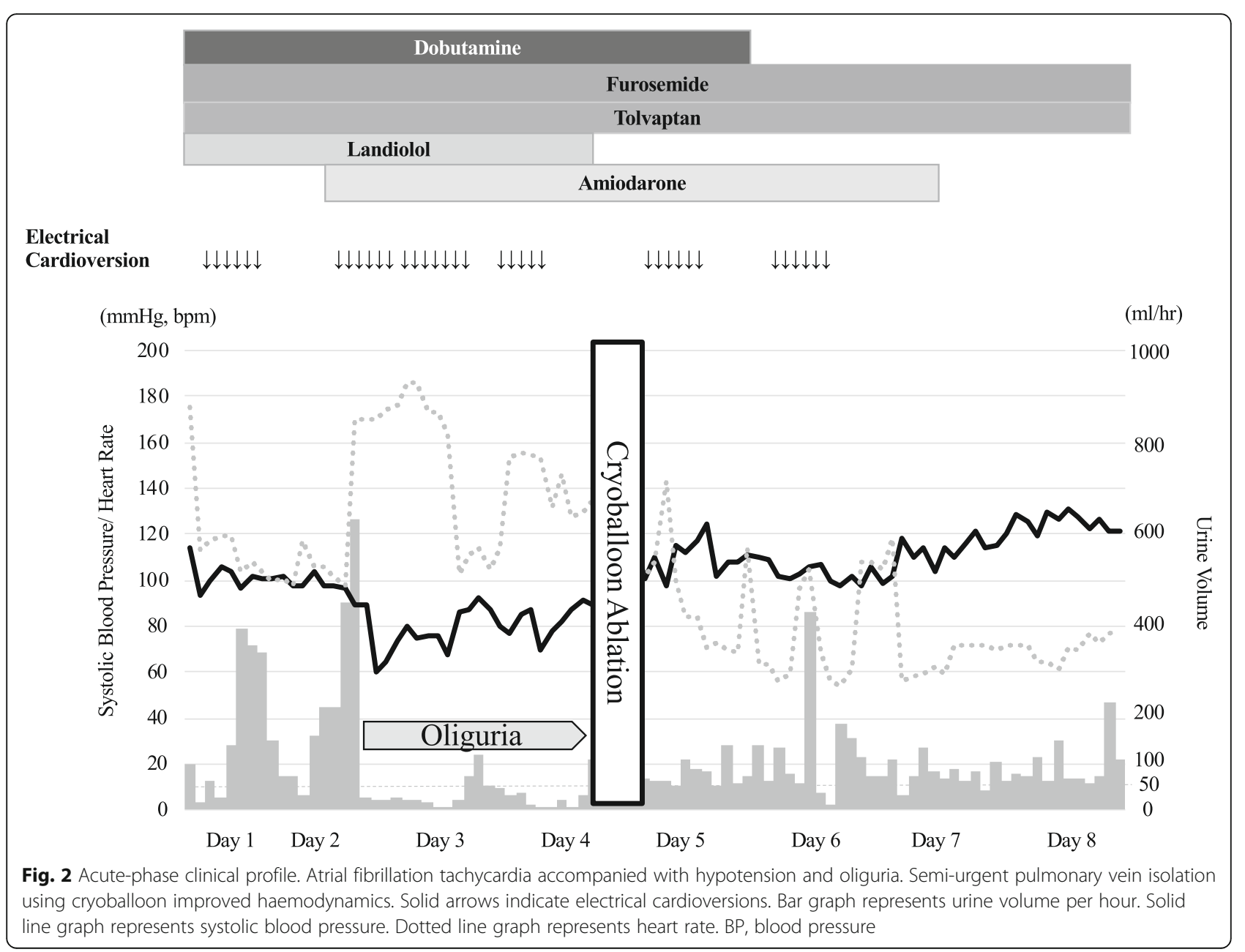


ventricular fibrillation as a complication of acute myocardial infarction [15]. This report also supported the benefit of semi-urgent rescue PVI on acute-phase rhythm control against haemodynamically unstable AF tachycardia. In addition, the present case had LOS-induced MOF and DIC in which organ perfusion flow was originally reduced due to left ventricular dysfunction and was additionally reduced by AF tachycardia storm [1]. Furthermore, especially in LOS-induced shock states, the importance of cardiac output increase and the resultant increase in blood pressure by rhythm control might be emphasised because of the following pathophysiology: (1) central shift of the circulating blood and organ perfusion in contrast flow reduction due to neurohormonal response [16], (2) change of the source of liver blood perfusion supply from the portal vein to the hepatic artery due to hepatic arterial buffer reaction [17], and (3) dependency of renal blood perfusion on cardiac output and on blood pressure in hypotension [18]. Together with these considerations, we thought that the acute-phase rhythm control by semi-urgent PVI would have a certain role in LOS-induced MOF due to AF tachycardia, at least in elimination as aggravation factor, and would enable focus on the intensive care of original diseases and disorders.

We chose the cryoballoon ablation for semi-urgent rescue PVI in the present case. Cryoballoon ablation is a balloon-based ablation system using cryoenergy. In recent years, cryoballoon ablation has become the most effective alternative approach to radiofrequency catheter ablation showing non-inferiority to radiofrequency catheter ablation in freedom from AF/AT recurrence and overall safety [7-9]. Several clinical studies showed shorter procedure time in cryoballoon ablation than that in radiofrequency catheter ablation [7, 9]. An animal study showed that cryoballoon ablation has lower incidence of thrombus formation than radiofrequency catheter ablation [12]. The shorter procedure time may suppose lesser load in intensive care setting. The lower incidence of thrombus formation may be favourable in intensive care setting accompanied with DIC. Although the significance of rescue cryoballoon ablation is unknown, we considered that cryoballoon ablation could be a favourable tool when we were required to perform semiurgent rescue PVI, like in the present case. On the contrary, balloon-based ablation including cryoballoon ablation generally uses additional amount of contrast medium. We might consider performing the balloon-based ablation including cryoballoon ablation without left atriography for the patient with acute kidney injury, like in the present case. At the same time, we would like to emphasise the importance of careful and timely assessment of the benefit and risk of semi-urgent rescue PVI using cryoballoon because semi-urgent PVI using cryoballoon could be a complex procedure for a complex case.
In conclusion, acute-phase rhythm control by semiurgent PVI using cryoballoon might be a considerable treatment option in patients with haemodynamically unstable AF tachycardia which is refractory to cardioversion and drug therapy and accompanied with LOS and LOS-induced MOF with DIC.

\section{Acknowledgements \\ We would like to thank Editage (www.editage.com) for English language editing. \\ Authors' contributions \\ TK (corresponding author) was involved in the clinical decision making and drafted the manuscript. YA revised the manuscript critically for important intellectual content and gave final approval of the version to be published. RM, FM, and KS performed the cryoballoon ablation. All authors, including $\mathrm{KK}, \mathrm{MS}, \mathrm{KO}, \mathrm{YM}, \mathrm{MY}$, and $\mathrm{Kl}$, have read and approved the final manuscript.}

\section{Funding}

We received no external funding for the study of this case or publication of this case report.

\section{Availability of data and materials}

The datasets used and/or analysed during the current study are available from the corresponding author upon reasonable request.

Ethics approval and consent to participate

Not applicable.

\section{Consent for publication}

Written informed consent was obtained from the patient and the patient's spouse for the publication of this case report and accompanying images.

\section{Competing interests}

Dr. Ajiro received a fellowship from Medtronic Japan and research rewards from Medtronic Japan and St. Jude Medical (now Abbott Japan) for unrelated research. The other authors declare that they have no competing interests.

Received: 11 June 2020 Accepted: 25 August 2020

Published online: 11 September 2020

\section{References}

1. Anter $E_{1}$ Jessup $M$, Callans DJ. Atrial fibrillation and heart failure treatment considerations for a dual epidemic. Circulation. 2009;119:2516-25.

2. Calkins H, Hindricks G, Cappato R, Kim YH, Saad EB, Aguinaga L, et al. 2017 HRS/EHRA/ECAS/APHRS/SOLAECE expert consensus statement on catheter and surgical ablation of atrial fibrillation: executive summary. J Arrhythm. 2017;33:369-409.

3. Hsu LF, Jaïs P, Sanders P, Garrigue $S$, Hocini M, Sacher, et al. Catheter ablation for atrial fibrillation in congestive heart failure. N Engl J Med. 2004; 351:2373-83.

4. Trulock KM, Narayan SM, Piccini JP. Rhythm control in heart failure patients with atrial fibrillation contemporary challenges including the role of ablation. J Am Coll Cardiol. 2014;64:710-21.

5. Marrouche NF, Brachmann J, Andresen D, Siebels J, Boersma L, Jordaens L, et al. Catheter ablation for atrial fibrillation with heart failure. N Engl J Med. 2018;378:417-27.

6. Al Halabi S, Qintar M, Hussein A, Alraies MC, Jones DG, Wong T, et al. Catheter ablation for atrial fibrillation in heart failure patients: a meta-analysis of randomized controlled trials. JACC Clin Electrophysiol. 2015;1:200-9.

7. Kuck KH, Brugada J, Fürnkranz A, Metzner A, Ouyang F, Chun KR, et al. Cryoballoon or radiofrequency ablation for paroxysmal atrial fibrillation. $\mathrm{N}$ Engl J Med. 2016;374:2235-45.

8. Schmidt M, Dorwarth U, Andresen D, Brachmann J, Kuck KH, Kuniss M, et al. Cryoballoon versus RF ablation in paroxysmal atrial fibrillation: results from the German ablation registry. J Cardiovasc Electrophysiol. 2014;25:1-7.

9. Aryana A, Singh SM, Kowalski M, Pujara DK, Cohen Al, Singh SK, et al. Acute and long-term outcomes of catheter ablation of atrial fibrillation using the 
second-generation cryoballoon versus open-irrigated radiofrequency: a multicenter experience. J Cardiovasc Electrophysiol. 2015;26:832-9.

10. Asakura H, Takahashi H, Uchiyama T, Eguchi Y, Okamoto K, Kawasugi K, et al. Proposal for new diagnostic criteria for DIC from the Japanese society on thrombosis and hemostasis. Thromb J. 2016;14:42.

11. KDIGO. Clinical practice guideline for acute kidney injury. Kidney Int Suppl. 2012;2:1-138

12. Khairy P, Chauvet P, Lehmann J, Lambert J, Macle L, Tanguay JF, et al. Lower incidence of thrombus formation with cryoenergy versus radiofrequency catheter ablation. Circulation. 2003;107:2045-50.

13. Haïssaguerre $M$, Jaïs $P$, Shah $D C$, Akahashi A, Hocini M, Quiniou G, et al. Spontaneous initiation of atrial fibrillation by ectopic beats originating in the pulmonary veins. N Engl J Med. 1998;339:659-66.

14. Yamane T, Date T, Kanzaki Y, Inada K, Matsuo S, Shibayama K, et al. Segmental pulmonary vein antrum isolation using the "large-size" lasso catheter in patients with atrial fibrillation. Circ J. 2007;71:753-60.

15. Morishima I, Sone T, Tsuboi H, Mukawa H. Rescue pulmonary vein isolation for hemodynamically unstable atrial fibrillation storm in a patient with an acute extensive myocardial infarction. BMC Cardiovasc Disord. 2012;12:110

16. Zelis R, Flain SF. Alteration in vasomotor tone in congestive heart failure. Prog Cardiovasc Dis. 1982;24:437-59.

17. Lautt WW. Mechanism and role of intrinsic regulation of hepatic arterial blood flow: hepatic arterial buffer response. Am J Phys. 1985;249:G549-56.

18. Rangaswami J, Bhalla V, Blair JEA, Chang Tl, Costa S, Lentine KL, et al. Cardiorenal syndrome: classification, pathophysiology, diagnosis, and treatment strategies: a scientific statement from the American Heart Association. Circulation. 2019;139:e840-e78.

\section{Publisher's Note}

Springer Nature remains neutral with regard to jurisdictional claims in published maps and institutional affiliations.

Ready to submit your research? Choose BMC and benefit from:

- fast, convenient online submission

- thorough peer review by experienced researchers in your field

- rapid publication on acceptance

- support for research data, including large and complex data types

- gold Open Access which fosters wider collaboration and increased citations

- maximum visibility for your research: over $100 \mathrm{M}$ website views per year

At $\mathrm{BMC}$, research is always in progress.

Learn more biomedcentral.com/submissions 